\title{
Effect of cobb angle and BMI on scoliosis surgery recovery in adolescent idiopathic scoliosis patients
}

\author{
Abdulmonem Alsiddiky ${ }^{1}$, Abdullatef Alrashoudi², Ahmed AL Sayed², Albaraa Aljerian², Abeer Aljomiah² \\ ${ }^{1}$ Associate Professor, Research chair of Spinal Deformities, College of Medicine,King Saud University, Riyadh, ${ }^{2}$ Medical Intern, College of \\ Medicine, King Saud University, Riyadh
}

\begin{abstract}
Aims and Objectives: To assess the effect of Cobb angle and body mass index (BMI) on surgery recovery outcomes in Adolescent Idiopathic Scoliosis (AIS) patients. Materials and Methods: A retrospective study of 79 Adolescent Idiopathic Scoliosis patients who underwent corrective operations, at King Khaled University Hospital, Riyadh, Saudi Arabia was carried out in 2014. The patients were divided according into Cobb's angle (mild, moderate and severe) and as per weight (healthy weight and overweight). Data of preoperative, intra-operative and postoperative information as well as measures of dailyliving activities were collected. Results: The mean age of 79 patients (70females) was 16.6 years. Cobb angle assessment was mild in $41(51.9 \%)$, moderate in $24(30.4 \%)$ and $14(17.7 \%)$ were severe. Mean surgery and recovery time of patients is statistically significantly different in relation to severity of Cobb's angle (mild, moderate and severe). There is highly statistically significant difference in mean ranks of days taken for different measures of daily living in relation to severity of Cobb's angle. The mean values of body mass index have significantly reduced after surgery, when compared with mean values at before surgery. The mean surgery time was statistically significantly higher in over weight patients ( 5.33 hours). Conclusions: The results show an effect of severity of Cobb angle on recovery measures of patients with AIS, but no effect of BMI. BMI was corrected in these patients after surgery. Prospective studies are required to assess clinical outcomes of post-operative surgery, satisfaction of patients towards their body image and quality of life.
\end{abstract}

Access this article online

Website:

http://nepjol.info/index.php/AJMS

DOI: 10.3126/ajms.v7i2.12904

E-ISSN: 2091-0576

P-ISSN: 2467-9100

Key words: Scoliosis, Cobb angle and Body mass index

\section{INTRODUCTION}

The origin of the term "scoliosis" dates back to Ancient Greece, which simply means crooked. ${ }^{1}$ The definition of the condition has become more advanced with progress in medicine and contemporary authors define scoliosis as a deformity of the spine where one or more lateral curvatures deviate from the midline with respect to the coronal plane; ${ }^{2}$ lateral deviation of the spine is a characteristic of the condition, but the three-dimensional deformation results in morphologic changes to the trunk and rib cage. ${ }^{3}$ Scoliosis is typically diagnosed based on the spinal angulation on the posterior-anterior radiograph: angulation 10 degrees or greater with rotation is indicative of scoliosis. ${ }^{4}$ This angle is also known as the Cobb angle. ${ }^{4}$ The etiologies of scoliosis vary and are classified broadly as idiopathic, congenital, neuromuscular, syndrome-related or scoliosis due to secondary reasons (e.g. tumors, trauma or infections). ${ }^{3}$ The most common type of disease is idiopathic scoliosis, which constitutes the vast majority of cases encountered in clinical practice. ${ }^{5}$ Scoliosis is further subdivided according to the age of onset to infantile, juvenile, and adolescent and adulthood scoliosis. ${ }^{6}$ Of these, the adolescent age group ismost commonly afflicted and is defined as the onset of scoliosis between the age of 10 years and the point of skeletal maturity. ${ }^{2}$ Adolescent idiopathic scoliosis (AIS) is therefore an important clinical problem, with ten percent of cases requiring medical intervention due to eventual curvature progression. ${ }^{7}$ Surgical intervention for 
AIS is commonplace once the curvature of the spine is over 40 degrees and aims to correct the deformity and prevent further deformation of the spine. ${ }^{7}$ The surgical outcomes in this age group are generally good, with few long-term side effects and relatively low failure rates of the procedure. ${ }^{8}$ However, there are reported cases of acute and deep infection, neurological damage, pseud arthrosis and mortality following surgical intervention. ${ }^{9}$ Hence, it is important to consider the factors that may contribute to unsuccessful surgical outcomes in this group. ${ }^{9}$ One potential factor is the body mass index (BMI) of the patient, which has been shown to correlate with adverse outcomes and the severity of Cobb angle subsequent patient recovery. Theobjectives of this study are to assess the impact of BMI and severity of Cobb angle on surgical outcomes in patients with AIS.

\section{MATERIALS \& METHODS}

This retrospective cohort study was performed at King Khalid University Hospital (KKUH) in Riyadh, Saudi Arabia. Data was collected from the files of 156 patients who underwent scoliosis corrective surgery done during Jan 2007 to Jan 2012, for which only 79 fell under our study's inclusion criteria: Cobb's angle between 40-115 degrees, aged between 10-40 years at the time of operation, provisional diagnosis of AdolescentIdiopathic Scoliosis (AIS), patients who underwent only one scoliosis corrective surgery and patients who underwent Posterior release and instrumentation type of operation. Data was collected in the period from Jan 2014 to Feb 2014 which includes basic demographic information and information of preoperatively Cobb's angle, Haemoglobin level, Bone Mineral Density (BMD), Intra-operatively length of surgery, blood loss, requirement of blood transfusion, Haemoglobin levels. Postoperatively (early and late) time spent in the recovery room, Nil Per Oral (NPO) state, time to mobilization, time to discharge, long term outcomes of surgery measures (number of days taken for measuring activities of daily living) which were collected by phone interviews. Height and weight were also collected from each file and BMI was evaluated before and after undergoing the operation, and divide according to world health organization to, a BMI lower than 24.9 considered as a persons with healthy weight, those with a BMI greater than 25 were considered as persons with overweight. Patient files and an electronic database were used to obtain preoperative, radiographic, and surgical outcomes of patients surgically treated for AIS by posterior release and instrumentation from 2007 to 2012. Two radiographic variables were collected preoperatively, including Cobb's angels from spine $\mathrm{X}$-rays and Bone mineral density (BMD) from the DEXA scans.

\section{Statistical analysis}

Data were analyzed using SPSS version 21.0 software (Chicago, IL, USA). Descriptive statistics (mean, standard deviation, median, inter quartile range and percentages) were used to describe the quantitative and categorical variables. Student's independent t-test and paired t-test were used to compare the mean values of two independent samples and paired samples (before and after surgery) of quantitative study variables. One-way analysis of variance was used to compare the mean values of quantitative variables across the study variable of more than two categories. Non-parametric statistical tests (Mann-Whitney $\mathrm{U}$-test \&Kruskal Wallis test) were used to compare the mean ranks of study variables, which follow skewed distribution. A p-value of $<0.05$ and $95 \%$ confidence intervals were used to report the statistical significance and precision of results.

\section{Ethical considerations}

As this was a retrospective analysis patient permission was not considered appropriate, hence all data was used following Board ethical approval. All information remains anonymous during the analysis stage, with encrypted files used for data storage; only the author had access to these files during the study period.

\section{RESULTS}

Of the 79 patients, there were $70(88.6 \%)$ females. The mean (standard deviation) age was 16.6 (5.1) years, body mass index 19.78 (4.6) and Cobb angle was 59.8 (18.3). The number of patients with cobb angle between 40 to 60 (mild) were 41 (51.9\%), between 61 to 75 (moderate) were $24(30.4 \%)$ and above 75 (severe) were 14 (17.7\%) patients. Patients with body mass index less than 24.9 (healthy weight) were $67(84.8 \%)$ and with body mass index greater than 25 (over weight) were $12(15.2 \%)$ patients.

\section{Severity of Cobb's angle and its effect}

There mean (sd.,) surgery time and recovery time of these AIS patients was 4.5 (1.3) and 2.1 (1.4) hours. There is statistically significant difference in the mean surgery time and recovery time of patients in relation to the severity of Cobb's angle (mild, moderate and severe). That is the mean surgery time and recovery time is significantly higher (5.21 hours and 3.66 hours) in patients with severe cobb's angle when compared with surgery time and recovery time of patients with mild and moderate cobb's angle (4.25, $4.69 ; 1.54,2.03$ hours). Also there is highly statistically significant difference in the mean ranks of days taken for different measures of daily living in relation to the severity of Cobb's angle. The patients with moderate and severe cobb's angle have taken to statistically significantly 
higher number of days to perform the measures (feeding, grooming, dressing, bathing, toilet use, transfers, mobility and stairs climbing) when compared patients with mild cobb's angle. Also the number of days for wound healing in patients with severe Cobb's angle was significantly higher when compared patients with moderate and mild Cobb's angle. (Table 1)

\section{Body mass index and its effect}

The body mass index was corrected in these AIS patients after surgery, in which the comparison of mean values of body mass index indicates statistically significant difference at all the three levels of severity of Cobb's angle (mild, moderate and severe). The mean values of body mass index have significantly reduced after surgery, when compared with the mean values at before surgery (Table 2).The comparison of mean ranks of number of days taken to perform daily activities, between healthy weight and overweight patients shows no significant difference for feeding, grooming, dressing, bathing, toilet use, transfers, mobility and stairs climbing. The mean surgery time was statistically significantly higher in over weight patients (5.33 hours) when compared with healthy weight patients (4.43 hours)(Table 3$)$.

\begin{tabular}{|c|c|c|c|c|}
\hline \multirow[t]{2}{*}{ Variables } & \multicolumn{3}{|c|}{ Cobb's angle } & \multirow[t]{2}{*}{$p$-value } \\
\hline & Mild & Moderate & Severe & \\
\hline \multicolumn{5}{|l|}{ Mean (Sd.,) } \\
\hline $\begin{array}{l}\text { Surgery time } \\
\text { (in hours) }\end{array}$ & $4.25(1.34)$ & $4.69(1.26)$ & $5.21(0.89)$ & 0.044 \\
\hline \multirow[t]{2}{*}{$\begin{array}{l}\text { Recovery time } \\
\text { (in hours) }\end{array}$} & $1.54(0.85)$ & $2.03(1.57)$ & $3.66(1.05)$ & $<0.0001$ \\
\hline & & & & p-value* \\
\hline \multicolumn{5}{|l|}{ Median (IQ) } \\
\hline Wound healing & $3.5(3.8)$ & $4(3)$ & $7(1.5)$ & $<0.0001$ \\
\hline Feeding & $7(16)$ & $14(14)$ & $21.5(12)$ & 0.001 \\
\hline Grooming & $14(7)$ & $21(7)$ & $21(14)$ & 0.005 \\
\hline Dressing & $19(13)$ & $28(19)$ & $28(28)$ & 0.004 \\
\hline Bathing & $18(13)$ & $30(21)$ & $30(28)$ & $<0.0001$ \\
\hline Toilet use & $14(14)$ & $21(7)$ & $25(10)$ & 0.002 \\
\hline Transfers & $16(7)$ & $21(7)$ & $24.5(15)$ & 0.022 \\
\hline Mobility & $98(75)$ & $140(208)$ & 180 (177) & 0.004 \\
\hline Stairs & $26.5(10)$ & $34.6(21)$ & $29(22)$ & 0.281 \\
\hline
\end{tabular}

\section{DISCUSSION}

This study represents one of the first attempts to describe the impact of Cobb angle and BMI on patients with AIS in terms of post-surgical recovery, defined by numerous characteristics. Recovery was considered from an intra-operative perspective, post-operative and during subsequent patient follow-up to provide a comprehensive review of this process. Overall, the findings indicate that there is a strong relationship between severity of Cobb angle and different measures of daily activities of living. Specific features noted to be closely associated with severity of Cobb angle found were the surgical time period (intraoperative) and recovery time. Also the change in BMI at three severity levels of Cobb angle (mild, moderate \& sever) was observed in this study. Watanabe et al. (2007) illustrated that both values BMI and Cobb angle were correlated in a population with scoliosis. ${ }^{10}$ However, this was not a population with AIS and therefore there may be key differences between the pathogenesis of scoliotic changes and BMI in this group. Recent data has indicated that the relationship between Cobb angle and weight is significant in a population with AIS. ${ }^{11}$ However, this was a study population comprised of males only and the clinical scenario may differ in female patients with AIS. Therefore, the data presented here supports the relationship between increased BMI and an increased Cobb angle in female patients with AIS. This may reflect an exacerbating effect of obesity on spinal structure and should inform clinicians as to the importance of weight management in order to prevent further deformity in this population. ${ }^{12}$

The significant difference of time spent in surgery across the levels of Cobb's angle severity and also between the patients with healthy weight and overweight was observed and concurred with the published data. ${ }^{5,6}$ Peng et al. (2009) conducted an analysis of patients undergoing anterior spinal surgery and noted that the length of time of surgery was generally higher for obese patients, compared to those who had a lower BMI. ${ }^{7}$ This has been supported in other surgical scenarios, where patient obesity can have an impact on visualisation of the anatomy, anaesthetic considerations and an increased likelihood of mechanical difficulties during surgical manipulation of tissue. ${ }^{8}$ However, an older study in patients undergoing spinal surgery noted that there was no significant difference between the lengths of

\begin{tabular}{|c|c|c|c|c|c|}
\hline Cobb angle & BMI before surgery & BMI after surgery & Difference of mean & $p$-value & $95 \%$ confidence interval for difference of mean \\
\hline Mild & $20.06(4.5)$ & $18.84(4.4)$ & 1.22 & $<0.001$ & $(0.90,1.54)$ \\
\hline Moderate & $20.60(5.0)$ & $19.22(4.6)$ & 1.38 & $<0.001$ & $(1,05,1.71)$ \\
\hline Severe & $17.56(3.8)$ & $15.82(3.5)$ & 1.74 & $<0.001$ & $(1.26,2.21)$ \\
\hline
\end{tabular}




\begin{tabular}{|c|c|c|c|}
\hline Variables & $\begin{array}{l}\text { Healthy } \\
\text { weight }\end{array}$ & $\begin{array}{c}\text { Over } \\
\text { weight }\end{array}$ & p-value \\
\hline \multicolumn{4}{|l|}{ Mean (Sd.,) } \\
\hline Surgery time (in hours) & $4.43(1.22)$ & $5.33(1.47)$ & 0.030 \\
\hline \multirow[t]{2}{*}{ Recovery time (in hours) } & $2.17(1.44)$ & $1.45(0.69)$ & 0.108 \\
\hline & \multicolumn{3}{|r|}{ p-value* } \\
\hline \multicolumn{4}{|l|}{ Median (IQ) } \\
\hline Blood loss & $800(600)$ & $1200(500)$ & 0.022 \\
\hline Blood transfusion & $2(1)$ & $7(5)$ & 0.004 \\
\hline Wound healing & $5(3.5)$ & $4(2)$ & 0.414 \\
\hline Feeding & $14(14)$ & $7(9)$ & 0.217 \\
\hline Grooming & $14(9)$ & $14(11)$ & 0.709 \\
\hline Dressing & $21(16)$ & $21(15)$ & 0.530 \\
\hline Bathing & $21(28)$ & $21(14)$ & 0.272 \\
\hline Toilet use & $21(14)$ & $17(13)$ & 0.345 \\
\hline Transfers & $21(7)$ & $18(7)$ & 0.419 \\
\hline Mobility & 112 (113) & $104(91)$ & 0.181 \\
\hline Stairs & $28(14)$ & $25(21)$ & 0.723 \\
\hline
\end{tabular}

surgery in either obese or non-obese patients, indicating that this may not be a very significant feature in the surgical process. ${ }^{9}$ Our findings also suggested that overweight was associated with higher operating times.

One of the most surprising aspects of this study is that overweight did not appear to have a profound effect on intra-or post-operative recovery times and did not seem to affect patient morbidity as a result. Other studies have noted that BMI can influence the outcome of scoliosis surgery and demonstrated that patients with elevated BMI prior to surgery tended to experience greater postoperative pain and usually had more severe surgical disease. ${ }^{8,9}$ Other data indicates that spinal surgery is more likely to be associated with post-operative infection where BMI is elevated, regardless of the aetiology of the disease. ${ }^{9}$ These studies were all relevant to adult, rather than adolescent populations, and the rate of comorbidities and complications was relatively high compared with those seen in adolescent data. ${ }^{13,14}$ Therefore, it is difficult to surmise if these are relevant to the adolescent population with AIS.

Upasani et al. (2008) conducted a similar study to that presented in this paper, where they explored the impact of obesity on surgical outcomes in patients with AIS, with a stronger focus on the success of surgical intervention and the rate of implant failure/revision required. ${ }^{12}$ The authors found that BMI was associated with thoracic kyphosis prior to surgical intervention, mimicking the findings presented here, and similarly noted that BMI did not have a profound effect on perioperative or postoperative morbidity or mortality. This study used a population of 204 patients aged between 12 and 16 years old, reflecting a larger sample size than the current study, although the sample size was still relatively small. However, despite the potential for a small sample size to result in poor rigor in data analysis, the authors suggested that adverse outcomes following scoliosis surgery might be related to other squeal that are present in the adult population, but have not had time to develop in this younger age group. ${ }^{12}$ The findings of the current study support the work of Upasani et al. and it is an interesting point to note that the general improved health of the adolescent population may minimise the impact of obesity of surgical outcome. Larger studies would be needed to explore these findings in greater detail, particularly as obesity is dramatically increasing in this age group and has an earlier age of onset, potentially leading to increased development of adverse squeal in adolescence. ${ }^{15,16}$

The other finding worth mentioning from this study is the significant difference of requirement of blood transfusion between health weight and overweight during surgery. The physiological reason for increased blood loss during surgery may be associated with the decreased venous return found in obese patients due to intrathoracic pressure increases from abdominal content movement. ${ }^{17}$ Hence, venous congestion may occur increasing relative blood loss during surgery. ${ }^{18}$ However, despite this physiological mechanism, data has suggested that estimated blood loss, even when surgical time is increased, may not differ significantly from that expected in the non-obese population. ${ }^{5,19}$ However, recent data has indicated that there may be an increased transfusion need in overweight patients during thoracic and lumbar fusion surgery, which also correlated with an increased likelihood of discharge to assisted living facilities. ${ }^{20}$ Hence, this study identifies that transfusion need may be elevated in the overweight patients and that this can have significant effects on post-operative outcome. This study did not assess the AIS population; however, and therefore it is unclear if these findings are transferrable. Although the data presented here highlights the potential for an increased transfusion need in the obese population with AIS this was not found to be associated with short-term or long-term outcomes, lessening the clinical significance of this feature. However, it is important that patients with an elevated body weight are thoroughly assess prior to surgery and that excessive blood loss is accounted for so that suitable replacement can be delivered during surgery, thus preventing any complications from occurring. ${ }^{17}$ This study did not use the Barthel index of activities of daily living score before and after surgery due to the retrospective study of surgeries, but the performance of activities of daily living was recorded by interviews after surgery. The results indicate significant difference in the number of days taken by the patients for their daily activities, who had serve Cobb angle when compared with 
mild and moderate Cobb angle. But BMI does not have any effect on the number of days for daily activities.

There are several limitations to the data collection process and the analysis of results that should also be considered when placing these findings into a clinical context. Firstly, there were very few overweight patients recruited in this study and therefore variations in BMI tended to occur across the underweight-to-overweight range. This means that the effect of a grossly elevated BMI was underestimated in this sample and hence the consequences of more severe obesity may not be fully appreciated based on this study alone. In addition, the rate of complications and adverse outcomes was also low, diminishing the power of this study to identify an association between obesity and adverse clinical outcomes. This is most likely due to the age and relative health of the patients recruited and therefore would be difficult to avoid in future studies. Finally, determination of an association between variables does not necessarily indicate a causative effect and therefore caution should be maintained when attributing BMI to specific outcomes in surgery. Other factors that contribute to BMI, including dietary and lifestyle factors may influence both of these variables and hence causation is not referred to in this literature. ${ }^{21}$ Future studies should employ a controlled intervention methodology in order to determine the impact of obesity in the AIS population, which remains woefully understudied in this context. In addition, more suitable markers of functional outcome, appropriate to the adolescent age group may be needed, as bowel function, mobility and other markers of daily activity were generally unaffected in this study. ${ }^{22}$

Despite these limitations, a number of recommendations for practice can be formulated based on these findings. Firstly, there is a need to ensure that obese patients have been thoroughly assessed prior to surgery for AIS in order to ensure that the potential for increased blood loss is accounted for. Secondly, surgeons and anaesthetists should consider the need for extending operating time and facilities in this patient group in order to minimise complications and ensure venous return is optimised. In addition, primary care physicians and medical staff should be aware of the potential for obesity to exacerbate AIS and other spinal conditions, promoting lifestyle interventions and dietary interventions where appropriate in order to manage the condition. At present, there is insufficient data to recommend specific interventions to reduce postoperative complications and enhance quality of life in patients with AIS who are surgical candidates. However, future studies are needed to ensure that outcomes are optimised, regardless of BMI, and hence should be a priority for the future.

\section{CONCLUSION}

In summary, this study identified the effect of severity of Cobbangle and post-surgical outcomes and recovery in patients with AIS, but not the effect of BMI. And the effect of BMI, on the increased need for blood transfusion in patients with a higher BMI, has important implications for practice and may ultimately have an impact on patient outcome. The AIS group can be considered healthier than populations where spinal disease is prevalent in adults, due to a reduction in the accumulation of disease and comorbidities and therefore the power to detect an effect of BMI and other outcomes is lower in this population. Hence, future studies need to recruit larger numbers of patients to increase the ability to detect such effect. Furthermore, including an increased number of obese patients will provide greater information as to the specific impact of obesity, rather than smaller fluctuations in BMI. These studies are needed in order to optimise the care pathways for patients with AIs and obesity and ensure that post-surgical complications and revision rates can be reduced by appropriate peri-operative planning and management.

\section{Declaration}

The authors did not receive any funding towards this work and no conflict of interest.

\section{REFERENCES}

1. Lovett RW. The history of scoliosis. The Journal of Bone and Joint Surgery. 1913;11(1):54-62.

2. Nault ML, Allard P, Hinse S, Le Blanc R and Caron O. Relations between Standing Stability and Body Posture Parameters in Adolescent Idiopathic Scoliosis. Spine 2002; 27(17):1911-1917.

3. Janicki JA and Alman B. Scoliosis: Review of diagnosis and treatment. Paediatric Child Health 2007; 12(9):771-776.

4. Kane WJ. Scoliosis prevalence: a call for a statement of terms. Clinical Orthopedics1997; 126:43-46.

5. Roach JW. Adolescent idiopathic scoliosis. Orthopedic Clinics of North America 1999; 30(3):353-365.

6. Reamy BV and Slakey JB. Adolescent idiopathic scoliosis: review and current concepts. American Family Physician 2001; 64(1): 111-117.

7. Miller $\mathrm{NH}$. Cause and natural history of adolescent idiopathic scoliosis. Orthopaedic Clinics of North America 1999;30(3):343-352.

8. Bridwell $\mathrm{KH}$. Spinal instrumentation in the management of adolescent idiopathic scoliosis. Clinical Orthopaedics 1997; 335:64-72.

9. Lonstein JE. Adolescent idiopathic scoliosis. Lancet 1994;344:1407-1412.

10. Watanabe G, Kawaguchi S, Matsuyama T and Yamashita T. Correlation of scoliotic curvature with Z-score bone mineral density and body mass index in patients with osteogenesis imperfect. Spine 2007; 32(17): 488-494.

11. Oh CH, Yoon SH, Park HC, Park $\mathrm{CO}$ and Kim SY. A comparison 
of the somatometric measurements of adolescent males with and without idiopathic scoliosis.Journal of Spinal Disorders and Technology 2013; doi: 10.1097/BSD.0b013e31828af710

12. Upasani VV, Caltoum C, Petcharaporn M, Bastrom T, Pawelek J, Marks M, et al. Does obesity affect surgical outcomes in adolescent idiopathic scoliosis? Spine 2008; 33(3): 295-300.

13. Di Raimondo CV and Green NE. Brace-wear compliance in patients with adolescent idiopathic scoliosis. Journal of Pediatric Orthopedics 1988; 8:143-146.

14. Rigo M, Reiter $\mathrm{CH}$ and Weiss HR. Effect of conservative management on the prevalence of surgery in patients with adolescent idiopathic scoliosis. Pediatric Rehabilitation2003; 6(3-4): 209-214.

15. Weiss HR and Goodall D. The treatment of adolescent idiopathic scoliosis according to present evidence.European Journal of Physical Rehabilitation Medicine.2008; 44: 177-193.

16. Lenke LG. What's new in the surgical care of adolescent idiopathic scoliosis (AIS). ArgoSpine News and Journal 2012;24 (1-2): 62-66.
17. Graham EJ, Lenke LG, Lowe TG, Betz RR, Bridwell KH, Kong Y, et al. Prospective pulmonary function evaluation following open thoracotomy for anterior spinal fusion in adolescent idiopathic scoliosis. Spine 2000;25(18): 2319-2325.

18. Steib JP, Dumas R, Mitton D and Skalli W. Surgical correction of scoliosis by in situ contouring: a detorsion analysis. Spine 2004; 29(2): 193-199.

19. Lehman RA, Lenke LG, Keeler KA, Kim YJ, Buchowski JM, Cheh $\mathrm{G}$, et al. Operative treatment of adolescent idiopathic scoliosis with posterior pedicle screw-only constructs: Minimum three-year follow-up of one hundred fourteen cases. Spine 2008; 33(14): 1598-1604.

20. Weiss HR. Adolescent idiopathic scoliosis- case report of a patient with clinical deterioration after surgery. Patient Safety in Surgery 2007; $1: 7$.

21. Weinstein SL, Zavala DC and Ponseti IV. Idiopathic scoliosis: long-term follow-up and prognosis in untreated patients. Journal of Bone and Joint Surgery America 1981; 63:702-712.

22. Dubousset J, Herring JA and Shufflebarger $H$. The crankshaft phenomenon.Journal of Pediatric Orthopedics 1989; 9:541-550.

\section{Authors Contribution:}

AM- Design of the study and manuscript preparation; AL- Review of literature, data collection and helped in preparing first draft of manuscript;

AS- Data collection, data entry and statistical analysis; AA- Data collection, data entry and statistical analysis; AJ- Manuscript Editing and Review. 